The ASTROPHYSiCal Journal SuPPLEMENT SERIES, 90:765-774, 1994 February

(C) 1994. The American Astronomical Society. All rights reserved. Printed in U.S.A.

\title{
ACCELERATION FROM FIELD-ALIGNED POTENTIAL DROPS
}

\author{
Gerhard HAERENDEL \\ Max-Planck-Institut für extraterrestrische Physik, 85740 Garching, Germany \\ Received 1993 March 8; accepted 1993 June I
}

\begin{abstract}
Unstable field-aligned currents are seen as the origin of field-aligned potential drops. They convert energy stored in magnetic shear stresses into kinetic energy. A good fraction of this energy is carried by runaway electrons and ions out of the acceleration region. The paper emphasizes the analogy with mechanical fractures. Simple expressions for the energy conversion rate and the parallel potential drop are derived, the two being linked by the critical current density needed for instability. The origin of the currents (generator) lies mostly in a region remote from that of energy conversion (fracture zone). The transmission of shear stresses and energy from the generator plasma, where the primary forces are applied to the fracture zone is also considered. A closed set of relations allows quantitative evaluation of the energetic particle production efficiency. The decoupling of the plasma on either side of the fracture zone which allows fast stress relief is described in detail, as well as a stationary model for the Alfvén wave interaction between fracture zone and generator plasma. A simple concept of the nature of the anomalous resistivity generated by the unstable current leads to an expression for the magnetic diffusivity inside the fracture zone and an estimate of the latter's extent parallel to the magnetic field, whereas its width and length transverse to $\boldsymbol{B}$ follow from the macroscopic relations. Finally and as an example, the theory is applied to the problem of fast electron (and ion) acceleration well above $1 \mathrm{MeV}$ seen to occur in many solar flares. It is obvious that this process belongs to the most powerful production processes of high-energy particles in stellar magnetic fields.
\end{abstract}

Subject headings: acceleration of particles — MHD — plasmas

\section{INTRODUCTION}

Acceleration from magnetic field-aligned potential drops is known to occur at several $1000 \mathrm{~km}$ altitudes above auroral arcs (e.g., Mozer et al. 1977; Sharp, Johnson, \& Shelley 1977; Gorney et al. 1981). It is thus the closest realization of a powerful cosmic acceleration process, easy to probe, and, indeed, documented by a wealth of data (a good collection of papers with data from the Swedish Viking satellite can be found in the May issue [no. A5, vol. 95] of the J. Geophys. Res. 1990). The more one should be surprised that few attempts have been made to transfer the knowledge gained from the aurora to other cosmical situations. Electrostatic acceleration has received much attention in the context of pulsar magnetospheres (e.g., Goldreich \& Julian 1969) and is occasionally thought to occur in solar flares (e.g., Colgate 1978). An acceleration process so common to the benign plasma environment of the Earth can be rightly suspected to exist in any stellar magnetic field that is sufficiently agitated. It is, therefore, desirable to cast the essence of the auroral acceleration process into a set of physical relations that would allow quantitative application to other cosmical objects, provided enough information exists about the physical properties of the object.

Unfortunately, there are many competing theories of auroral acceleration (see Borovsky 1993). The perhaps most widely accepted one regards the field-aligned potential drop as a means of maintaining the continuity of auroral field-aligned currents against the mirror force acting on the energetic electrons provided they are the dominant current carriers (e.g.,
Knight 1973; Fridman \& Lemaire 1980; Lyons 1980). This theory has been found to be quite successful in explaining many features of the primary auroral particle distributions. A second class of theories attributes the existence of parallel electric fields to the appearance of anomalous resistivity in intense field-aligned currents (Haerendel 1980; Lysak \& Dum 1983 ). Field-aligned currents play the key role in both theories, but they differ in that the first one depends on hot magnetospheric electrons being the current carriers, whereas the second one deals with the currents as they are continued in the "cold" plasma of the topside ionosphere. Kindel \& Kennel (1971) found that it is here that a given field-aligned current becomes most easily unstable, excites microturbulence, and thereby creates an effective anomalous resistivity. This process has a critical current (or drift velocity) threshold, whereas the mirror effect does not. However, it so happens that the physical parameters of the lower magnetosphere and upper ionosphere are such that both effects can be (and probably are) realized simultaneously, but at different altitudes. The existence of sufficiently strong field-aligned current densities above the auroral zone has been frequently verified (Casserly \& Cloutier 1975; lijima \& Potemra 1976).

It can be shown that the relative importance of the two mentioned processes in creating field-aligned potential drops depends on the total plasma density in the sense that with increasing density the first class loses importance. In the environment of solar flares, for instance, it is entirely negligible. For this reason, I will not include the mirror process in the subsequent discussion of field-aligned potential drops. The pre- 
sented theory has evolved over more than 10 years, but so far it has been published exclusively in conference proceedings and the alike (Haerendel 1980, 1987, 1989).

\section{ENERGY RELEASE FROM SHEARED MAGNETIC FIELDS}

Field-aligned potential drops as a product of intense fieldaligned electric currents imply the existence of strongly sheared magnetic fields. Thus the ultimate cause of the related cosmic acceleration process and supplier of the energy expended there are the forces creating the magnetic shear. A prominent feature of this acceleration process is that energy supply and energy conversion (into particle beams) are physically separated. There is one plasma regime, mostly characterized by a high value of the plasma beta, that is, the ratio of gas and magnetic pressures $\left(\beta=8 \pi p / B^{2}\right)$, where some acting mechanical force, like friction, pressure gradient, or the inertial force, cannot be (fully) balanced by magnetic normal forces, but require the transport of shear stresses out of the interaction volume into another plasma regime where they are absorbed. The transport of shear stresses is effected by field-aligned currents. On their way to the stress absorption (or reflection) region, the currents may encounter conditions rendering them unstable, that is, $j_{\|}$may reach a threshold value, $j_{\text {crit }}$, for a powerful microinstability process. As a consequence, a region of high anomalous resistivity can develop sustaining large field-aligned potential drops. The plasma regime in which the conditions for this to occur are favorable is typically characterized by very low values of $\beta$ owing to the magnetic field concentration near the star. Concentrations of magnetic flux and of field-aligned currents go together. If, in addition, due to the presence of a cool plasma constituent, the thermal velocity is low and the plasma density not too high, instability can easily arise. Such conditions are most likely met at the top of a cool atmosphere/ionosphere (Kindel \& Kennel 1971).

Figure 1 is a sketch of such a cosmical situation. Shearing forces pointing out of the plane on the left-hand side $(\beta \cong 1$ plasma) generate currents which are transmitted along the

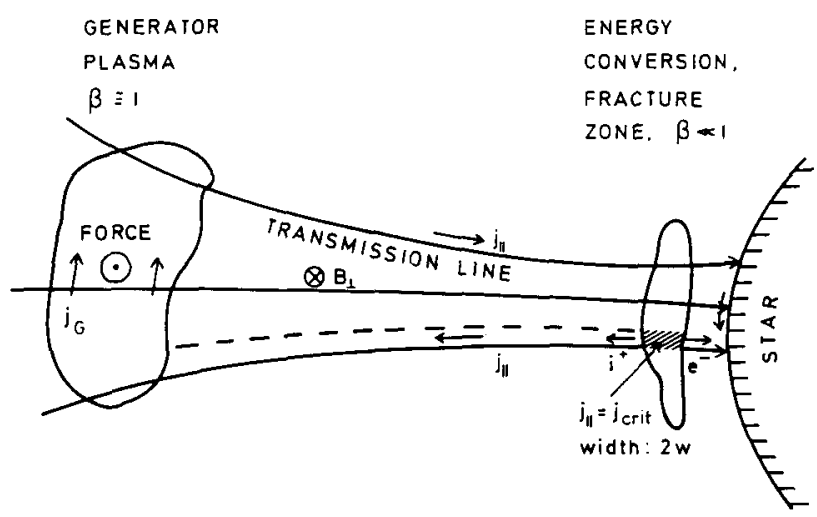

Fig. 1.-Schematic representation of magnetic configuration of the energy conversion process. Left: primary mechanical forces which apply the shear stresses; middle: stress (and energy) transmission region; right: current closure near star and a region of field-aligned current instability, where the energy conversion takes place. Beams of ions and electrons emerge from the conversion region or fracture zone. magnetic field toward the star. Somewhere in its outer atmosphere (to the right of the figure), not necessarily where the currents close, one of the two branches of $j_{\|}$may become unstable:

$$
j_{\|}=j_{\text {crit }}=e n_{c} c_{\text {crit }}
$$

The dominant cold plasma in this $\beta \ll 1$ regime has density, $n_{c}$, and electron temperature, $T_{e}$. We may scale $c_{\text {crit }}$ by the ionacoustic speed, $c_{s}=\sqrt{k T_{e} / m_{i}}$ :

$$
c_{\text {crit }}=f \cdot c_{s}
$$

The value of the scaling factor, $f$, depends on the prevailing instability which, in turn, depends on the ratio $T_{e} / T_{i}$ and on the composition (Papadopoulos 1977). We will not be specific on this point but note that $f$ should be of order 10 .

Figure 1 shows the current to be concentrated in a thin sheet of width, $2 w$. This concentration is an essential feature. Even for the aurora borealis it is not well understood what processes lead to such current concentrations. However, a number of nonlinear processes which may have such consequences come to mind, such as MHD instabilities, formation of X-type neutral lines (reconnection), or in case of the ionosphere, conductivity changes due to the closure of $j_{\|}$. In most cosmical applications, rather than predicting current densities, it should be easier to evaluate the magnitude of the total transverse magnetic field component, $\boldsymbol{B}_{\perp}$, generated by the shear forces. The change of $\boldsymbol{B}_{\perp}$ through the current sheet, $\Delta \boldsymbol{B}_{\perp}$, its width, $2 w$, and critical current density, $j_{\text {crit }}$, are related by Ampère's law:

$$
2 w=\frac{c\left|\Delta \boldsymbol{B}_{\perp}\right|}{4 \pi j_{\text {crit }}}
$$

$B_{\perp}$ can be guessed from the plasma dynamics in the generator plasma. The transmission of the stresses ( or $B_{\perp}$ ) from the generator toward the star will be treated below. Here $j_{\text {crit }}$ is an intrinsic property of the $\beta \ll 1$ plasma regime. So, $w$ is normally the least-known quantity in equation (3), with the exception of the auroral context, where the visible arc supplies directly the information.

Acceleration by field-aligned potential drops, $\phi_{\|}$, is a conversion process of free magnetic energy into kinetic energy of particle beams and heat. However, this process does not work simultaneously on the whole reservoir of free magnetic energy, but only on a boundary region where $j_{1}$ is sufficiently concentrated (eq. [1]) in order to develop anomalous resistivity due to some type of microturbulence and thus sustain a finite $\phi_{\|}$over a width, $2 w$. Besides converting magnetic energy into kinetic, $\phi_{1}(\neq 0)$ leads to a dynamic decoupling of plasmas pervaded by the same magnetic field lines. The frozen-in condition is drastically violated. Once a $\phi_{\|} \neq 0$ has been set up, the plasma on either side is free to move in the sense as to reduce the applied magnetic shear stresses.

This motion is no longer controlled by the line-tying to the star or its atmosphere, but by a simple relation between plasma velocity, $\boldsymbol{v}_{\perp}$, transverse to $B$ or the attendant electric field, $\boldsymbol{E}_{\perp}$, on the one hand, and the integrated sheet current, $\int j_{\|} d x_{\perp}$, or 
corresponding jump of $\boldsymbol{B}_{\perp}$, on the other. Two equivalent forms of this relation are

$$
E_{\perp}=R_{w} \int_{x_{\perp 1}}^{x_{\perp 2}} j_{l} d x_{\perp}
$$

and

$$
\boldsymbol{v}_{\perp}= \pm \frac{\Delta \boldsymbol{B}_{\perp}}{B_{0}} \cdot c_{\mathrm{A}}
$$

with

$$
R_{w}=\frac{4 \pi c_{\mathrm{A}}}{c^{2}}
$$

and

$$
c_{\mathrm{A}}=\frac{v_{\mathrm{A}}}{\sqrt{1+\left(\frac{v_{\mathrm{A}}}{c}\right)^{2}}} .
$$

Here $v_{\mathrm{A}}=B / \sqrt{4 \pi \rho}$ is the familiar Alfvén velocity and $\Delta \boldsymbol{B}_{\perp}=$ $(4 \pi / c) \int_{x_{\perp 1}}^{x_{\perp 2}} j_{1} d x_{\perp}$. In very strong magnetic fields like those of accreting neutron stars, $v_{\mathrm{A}}$ can exceed grossly the velocity of light, $c$. Alfvén waves can still exist, but they are dominated by displacement currents, and their phase velocity, $c_{\mathrm{A}}$, equals $c$. All the same, the stress relief velocity. $v_{\perp}$, may be largely subrelativistic. $R_{w}$ is the Alfvénic wave impedance. Equation (5) is the Walén relation.

The existence of a strong electric field, $\boldsymbol{E}_{\perp}$, in the current sheet is related to a Poynting flux, $S_{p}=c / 4 \pi \cdot E_{\perp} \times \Delta B_{\perp}$, which has a negative divergence in the acceleration region $\left(\phi_{\|} \neq 0\right)$. Another way of writing $S_{p}$ is in the form of transport of magnetic energy. The mean value of $S_{p}$ in the current sheet is

$$
S_{p}=\frac{1}{16} \times \frac{\left(\Delta B_{\perp}\right)^{2}}{4 \pi} c_{\mathrm{A}}
$$

The factor $\frac{1}{16}$ will be discussed in $\S 4$. It arises from the variation of $B_{\perp}\left(x_{\perp}\right)$ and $E_{\perp}\left(x_{\perp}\right)$ through the current sheet and from the fact that not all liberated magnetic energy is consumed in the $\phi_{1}$-region.

The decoupling of the plasma by $\phi_{\|} \neq 0$ is reminiscent of a mechanical fracture process since it initiates fast stress relief motions (Haerendel 1980). What happens in detail will be elaborated in the next section. We will use the designation "fracture zone" for the region of $\phi_{1} \neq 0$ and refer to the quantities in this region by subscript $F$.

The Poynting flux arriving above the fracture zone is converted into kinetic energy. We set $S_{p}$ from equation (8) equal to the energy conversion rate, $\dot{\varepsilon}_{\|}$, per unit cross section due to $\phi_{1} \neq 0$ :

$$
\dot{\varepsilon}_{\mathrm{h}}=j_{\mathrm{crit}} \cdot \phi_{\mathrm{I}}=\frac{\left(\Delta B_{\perp F}\right)^{2}}{64 \pi} \cdot c_{\mathrm{A} F} .
$$

Energy conversion does not equal dissipation. The dominant part of energy may appear in form of field-aligned particle beams, that is, runaway particles.

With help of equation (1) we gain from equation (9) an expression for the field-aligned potential drop, one of the main goals of the theory:

$$
e \phi_{\|}=\frac{c_{\mathrm{A} F} \cdot\left(\Delta B_{\perp F}\right)^{2} / 64 \pi}{n_{F} c_{\mathrm{crit}}} .
$$

The writing of the right-hand size lends itself to a simple interpretation:

$$
e \phi_{\|}=\frac{\text { E.M.ENERGY INFLOW }}{\text { PARTICLE OUTFLOW }}
$$

The ion and electrons emerging out of the acceleration region are a mix of accelerated runaway particles (dominantly electrons) and thermal particles. Energy-wise runaway particles seem to dominate, at least in the auroral realization of the process.

It remains to relate the energy flux (eq. [8]) at the fracture zone to that in the generator region where the primary shear forces act; that is, we have to deal with the "transmission line" in Figure 1. There are two cases, a stationary and a transient one. The first implies conservation of field-aligned current between generator and fracture zone:

$$
j_{\|} \cdot A \sim j_{\|} \cdot B^{-1}=\text { const }
$$

where $A$ is the cross section of a flux-tube, that is, $A \cdot B=$ const. The second case is based on conservation of energy flux. In WKB-approximation it can be expressed as

$$
\frac{\left(\Delta B_{\perp}\right)^{2}}{64 \pi} \cdot c_{\mathrm{A}} \cdot A=\text { const }
$$

With $\Delta B_{\perp} \sim j_{\|} \cdot w$ and $w \sim \sqrt{B}$, where $B$ is the total unperturbed field strength, we obtain the mapping factor, $m$, between generator and fracture zone:

$$
m=\frac{\Delta B_{\perp F}}{\Delta B_{\perp G}}=\frac{\left(\frac{B_{F}}{B_{G}}\right)^{1 / 2} \text { stationary case }}{\left(\frac{\rho_{F}}{\rho_{G}}\right)^{1 / 4} \text { transient case }}
$$

In equation ( $14 \mathrm{~b}), v_{\mathrm{A}}^{2} \ll c^{2}$ was assumed. In the opposite case $\left(v_{\mathrm{A}}^{2} \gg c^{2}\right)$ relation (14a) holds.

Equations (1)-(14) allow evaluation of the energy conversion by magnetic fractures for any stellar magnetic field. To this end, one must identify the primary shear forces and evaluate the possible magnitude of $B_{\perp G}$. Second, the most likely energy conversion region must be identified, that is, where along the current path $j_{\|}$is prone to become unstable. $B_{\perp G}$ can be mapped toward this region with help of equation (14). Equation (3) yields the typical width of an individual fracture zone. In most cases $w$ is likely to be very small compared with the transverse size of the stressed field region. Hence, an additional argument is needed as to how many fracture zones may 
exist at any time, corresponding to the number of parallel auroral arcs in the Earth's manifestation of this process. This number yields the total energy output. For distant cosmical objects, the reverse procedure is easier to apply. Knowledge of the total energy output, for example, through the resulting bremsstrahlung of the accelerated electrons, provides an estimate of the filling factor with fracture zones. Only if the latter turns out to be a very small number, can one accept the potential relevance of this process in generating the observed radiation.

Although all the tools for successful application of the theory have been assembled in this section, the reader may still feel uneasy about the true nature of the fracture process. The following two sections will be entirely devoted to an elaboration of what actually happens near the fracture zone $(\S 3)$ and between fracture zone and generator $(\S 4)$. In $\S 5$ we will address briefly some of the microphysical aspects of the parallel potential drops. Finally, in $\$ 6$ we will investigate the potential role of this process during solar flares.

\section{MAGNETIC FRACTURES}

Magnetic fractures or "breaking of field lines" (Haerendel 1980 ) are a realization of the reconnection process in very low beta plasmas. Plasma originally distributed along a certain flux-tube is found to become disconnected at the location of $\phi_{\|} \neq 0$. (For a more precise definition see Alfvén \& Fälthammar 1963, pp. 189-191.) However, in contrast to the more familiar reconnection process in high-beta plasmas, the topology of the reconnected field lines does not change (Vasyliunas 1975). The goal of this section is to clarify the plasma dynamics transverse to the magnetic field initiated by the insertion of $\phi \neq 0$ in the current system. We will look at it in three different projections. The discussion will be qualitative. The quantities are all contained in equations (1)-(14).

Figure $2 a$ is an illustration in a plane containing $B$ and $v_{\perp}$, the stress relief motion. Here we focus our attention on the neighborhood of the fracture zone. Its long-range interaction with the generator plasma is treated in the next section. Before entering the region of $\phi_{1} \neq 0$, which has a finite width, $2 w$, normal to the plane of projection, a stressed flux tube may have the direction indicated by the inclined solid lines. As soon as a finite $\phi_{\|}$is experienced somewhere along the field line, plasma above and below this region starts moving in the sense of reducing the magnetic shear stresses. Equation (5) gives the magnitude of the motion: a more detailed account will follow in $\S 4$. This motion is triggered by two Alfvénic wave fronts traveling away from the fracture zone with speed $c_{\mathrm{A}}$ (eq. [7]). The system typically has an inherent asymmetry (see Fig. 1). On one side of the fracture zone, the field is strongly anchored in the stellar atmosphere (or surface). Only short excursions of the flux tubes are allowed here which may be oscillatory or turbulent. On the other side, the side of the generator, normally the spatial scales extend much farther and $B$ decreases. This means that $c_{\mathrm{A}}$ decreases and that the timescales are much longer. Furthermore, the free magnetic energy content is considerably larger. Therefore, we will focus mainly on the events on this side from which the energy feeding the parallel acceleration process is dominantly derived.

After the free energy content of a flux tube has been exhausted, that is, after the shear stresses have been released, $\phi_{\|}$ will vanish and the frozen-in condition will apply again imposing strong coupling between generator plasma and stellar atmosphere. The overall magnetic configuration has hardly changed, except that $\boldsymbol{B}_{\perp}$, or the free magnetic energy, has been reduced. $B_{\perp}$ being small compared with $B_{0}(\beta \ll 1)$, two points found on the same field line at $t=0$, above and below the fracture zone, may become separated by large distances during the stress release process ( see $\S 4$ ).

The reader is asked to imagine a long rod anchored on one end and pulled in the perpendicular direction from the other end. Once it breaks somewhere at a weak point, it will initially undergo the same stress relief motions as one of the field lines sketched in Figure 2. On the timescale of elastic (Alfvénic) wave propagation along the rod (field line), oscillations will be set up. They will be treated in the next section, but the foundation of the expression "magnetic fracture" should already be obvious.

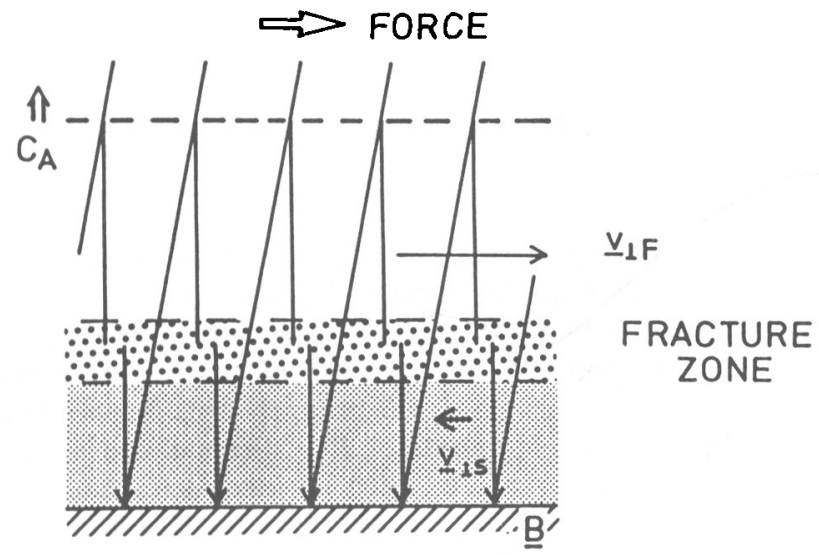

FIG. $2 a$

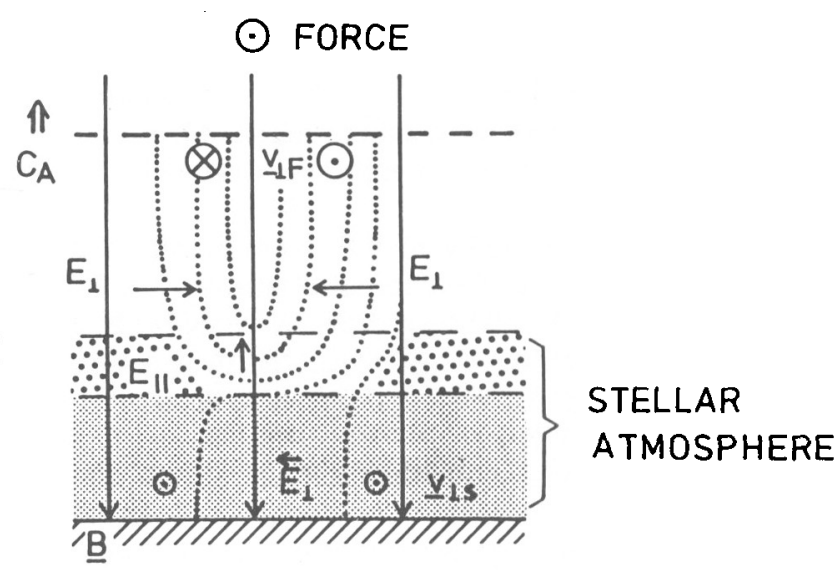

FIG. $2 b$

FIG. 2.- (a) Side view of plasma motions initiated by magnetic fractures, i.e., localized field-aligned potential drops. The inclined and vertical lines show the connectivity of plasma distributed along a field line as it changes through the stress relief motions, $v_{1}$, set up behind a propagating Alfvénic wave front. (b) Cross section of the same region perpendicular to the stress relief motions showing approximate equipotential contours and electric fields. 
The view of a cross section through the fracture zone perpendicular to $\boldsymbol{v}_{\perp}$, as given in Figure $2 b$, allows to sketch the typical equipotential contours related to $\phi_{\|}$. The contours depict the situation under neglect of the fast oscillations on the side of the star. Gurnett (1972), on the basis of particle spectra and electric fields measured above auroral arcs, proposed the existence of such $U$-shaped potential lines in the acceleration region. The underlying assumption that an electrostatic approximation is valid is by no means trivial. Overall the fracture process is an electromagnetic phenomenon involving emission and propagation of Alfvén waves. But the transit time of such a wave through the fracture zone is normally short compared to its lifetime. So a quasi-static, that is, electrostatic description is appropriate ( see $\S 5$ ).

Figure $2 b$ makes clear that $\phi_{\|} \neq 0$ implies strong electric fields on one side of the fracture zone which are not found on the other. Equation (4) gives their magnitude, equation (5) the corresponding transverse speed. A necessary consequence of the electrostatic nature of the electric field is that $\boldsymbol{E}_{\perp}$ on the leading side (right) is balanced by a nearly equally strong field (and transverse potential drop) on the trailing side (left):

$$
\phi_{\perp}=\int_{0}^{w} E_{\perp} d x_{\perp} \cong \phi_{\|}
$$

This trailing field and the corresponding reverse plasma motion are needed in order to observe the incompressibility of $\boldsymbol{B}$ which forbids accumulation of magnetic flux where $\beta \ll 1$. Of course, the response at the generator level $(\beta \cong 1)$ is entirely different.

Figure 3 depicts what happens along the trace of a fracture in a view parallel $\boldsymbol{B}$. On the side of the fracture zone adjacent to the interior of the stressed field region (upper half), the flow direction is in the sense of stress relief. On the outer side (lower half), the return flow enforced by the incompressibility of $B$ is seen. The magnetic shear stresses $\left(\boldsymbol{B}_{1}\right)$ fall off through the current sheet. Hence stress relief and return flows carry differently bent magnetic field lines. An erosion process is going on as the current sheet proceeds slowly into the stressed field region. Figure 3 shows this process in the reference frame of the fracture zone or current sheet. This progression of the fracture can continue as long as sufficiently stressed magnetic field exists in the interior of the current circuit.

The spatial extent, $\ell_{\perp}$, of a fracture in flow direction can be estimated by the time it takes a sheared flux tube to lose its free

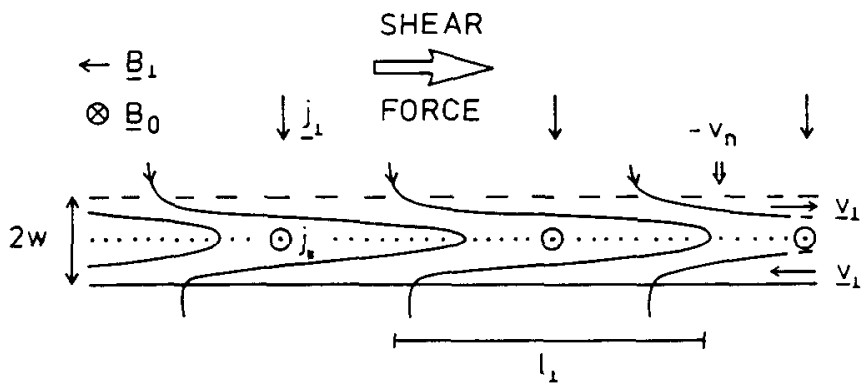

FIG. 3.-Plasma flow lines just above a fracture zone seen along the magnetic field, $\boldsymbol{B}_{0}$, and in the reference frame of the fracture zone propagating with normal velocity, $v_{n}$ energy content. This time scales with the travel time, $\tau_{\mathrm{A}}$, of a shear Alfvén wave between fracture zone and generator and return:

$$
\tau_{\mathrm{A}}=2 \int_{F}^{G} \frac{d \ell_{\|}}{c_{\mathrm{A}}\left(\ell_{\|}\right)} .
$$

The minimum length of a fracture is then

$$
\ell_{\perp}=v_{\perp} \cdot \tau_{\mathrm{A}}
$$

(In case of the aurora, $\ell_{\perp}$ is of order of a few $1000 \mathrm{~km}$, whereas the width of an arc is only of order $10 \mathrm{~km}$.) For maintenance of a quasi-stationary process, the current sheet must propagate into the stressed field region by the sheet thickness $w$ in a time of order $\tau_{\mathrm{A}}$. As will be demonstrated in the next section, this propagation speed, $v_{n}$, is approximately given by

$$
v_{n} \cong \frac{w}{2 \tau_{\mathrm{A}}}
$$

However, the mode of propagation may be oscillatory rather than smooth (Haerendel et al. 1993).

A key feature of the fracture process is the postulated spontaneity of the stress release and its progressive propagation into the stressed medium. The conditions for this to occur are not fully understood. Auroral observations indicate that it is not necessarily always the case. Often fractures may only last for a time of order $\tau_{\mathrm{A}}$. However, the typical property of auroral arcs to last much longer than $\tau_{\mathrm{A}}$ suggests the existence of quasistationary solutions. It also strengthens the analogy with fractures in solid bodies which propagate transverse to the stress direction because of stress concentration at their leading edge. The magnetic analogue of this is the maintenance of $j_{\|}=j_{\text {crit }}$ at the leading edge of the fracture zone.

The reader who is unfamiliar with auroral research may want some words on observational support for the outlined concept. I mention only four pieces of evidence out of a enormous wealth of data. Unfortunately, $\phi_{\|}$is usually distributed over several $1000 \mathrm{~km}$, and $\bar{E}_{\|}$is difficult to measure directly. However, particle distributions measured immediately above or below, but also inside the parallel potential drop reflect the experienced acceleration and allow in many cases a derivation of the magnitude of $\phi_{\|}$(Gorney et al. 1981). The transverse electric field, on the other hand, is strong and has been measured extensively. Mozer et al. (1977) were the first to measure the typical opposing $E_{\perp}$-components at about $1 R_{E}$ altitude and named them "paired electrostatic shocks" (Mozer et al. 1977). Another manifestation of these fields is the motion of auroral rays. If one observes auroral arcs along the magnetic field, the rays appear as "curls" (Hallinan \& Davis 1970). They move typically in stress relief direction at a speed corresponding to $\boldsymbol{v}_{\perp}$ above the fracture zone. However, in the low ionosphere where the visible aurora is created by electron impact, no corresponding plasma motion exists. The moving rays reflect a real plasma flow at high altitudes in and above the fracture zone thus bearing direct proof of the violation of the frozen-in condition. Often, albeit less strikingly visible, one can also find the counterflow expressed in the auroral emis- 
sions. Finally, a most impressive manifestation of the fracture process was achieved in a barium jet experiment in which a magnetic flux tube was "painted" by Ba ions visible over many $1000 \mathrm{~km}$ height extent. When an auroral arc developed at its "foot," the "painted" flux-tube was seen to split at about 5500 $\mathrm{km}$ altitude and to be quickly sheared into an extended sheet above this height while staying intact below it (Wescott et al. 1976). The reader is strongly encouraged to look at the printed TV pictures covering this experiment (Figs. 1 and 3 of Wescott et al.).

\section{INTERACTION WITH THE GENERATOR}

Current concentration in narrow sheets or filaments is a requirement for the appearance of $\phi_{\eta} \neq 0$ in a highly conductive plasma. Once it has been created, it may exist for extended periods by propagating into the volume of stressed magnetic field. Figure 4 shows what happens between generator plasma and star in a simplified geometry with straight field lines. It is assumed that only the upward current exceeds $j_{\text {crit }}$. Nothing dramatic happens with the region of downward current. There the magnetic field is perfectly frozen-in. The fracture zone initiates the energy release and thus constitutes the leading edge of

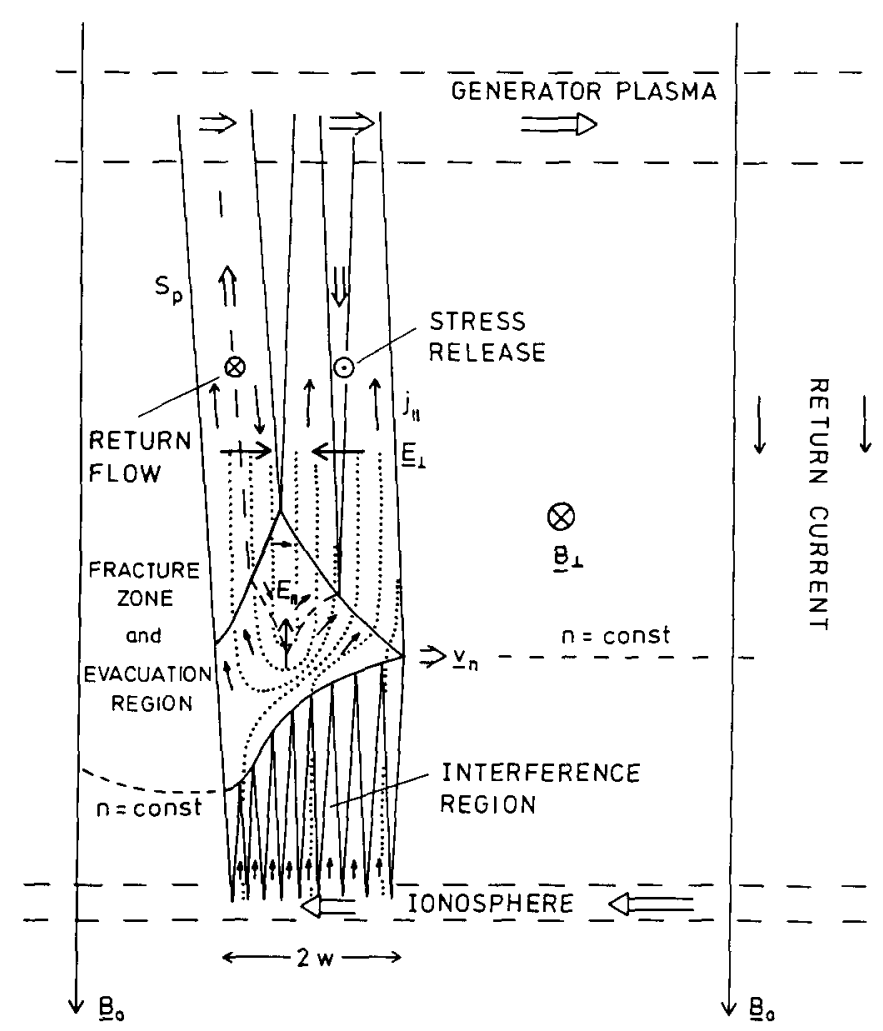

FIG. 4.-A qualitative stationary model of the long-range interaction of a parallel potential drop (fracture zone) with the generator of the current circuit, on one side, and the lower stellar atmosphere, on the other side. Slightly oblique Alfven waves are emitted from and attached to the slowly propagating fracture zone $\left(j_{\mathbf{l}}=j_{\text {crit }}\right)$. These waves switch on and off the primary stress relief motion (out of plane) and the return flow (into plane) imposed by the magnetic incompressibility. Reflection from the generator plasma and from the stellar atmosphere (ionosphere), which have grossly different timescales, lead to the depicted standing wave patterns. An evacuation process inside the fracture zone creates density depletions. the propagating current sheet. Attached to it are two standing Alfvén waves whose fronts form an extremely small angle, $\vartheta$, with respect to the field lines. The reason is the slow propagation speed, $v_{n}$, as compared with the Alfvén speed $c_{\mathrm{A}} . \vartheta$ can be derived from equations (16) and (18):

$$
\cot \vartheta=\frac{c_{\mathrm{A} F}}{v_{n}}=\frac{2}{w} \int_{F}^{G} \frac{c_{A F}}{c_{\mathrm{A}}\left(\ell_{\|}\right)} d \ell_{\|} .
$$

Not only is $\ell_{\|} \gg w$, but also $c_{\mathrm{A}}\left(\ell_{\|}\right)$normally decreases strongly toward the generator. Thus in looking at Figure 4 one must be aware that all transverse scales and angle $\vartheta$ are grossly exaggerated.

Because of the lateral propagation, $j_{\|}$is no longer strictly field-aligned. It acquires a small transverse component, $j_{\perp}=$ $j_{\|} \cdot \tan \vartheta$. By virtue of this component a Lorentz force is exerted on the plasma behind the wave front, and the plasma is set into motion with the speed:

$$
v_{\perp}\left(x_{\perp}\right)=\frac{c}{B} R_{w} \int_{x_{\perp 0}}^{x_{\perp}} j_{\sharp} d x_{\perp} .
$$

Equation (20) is just a differential version of equations (5) and (6).

The slightly oblique Alfvén wave hits the generator plasma after $\tau_{\mathrm{A}} / 2$. It is difficult to generalize what happens now. Nonlinear model calculations have not yet been performed. All the same, a few conclusions may be drawn. The mechanical forces acting on the generator plasma are in equilibrium with the magnetic shear stresses before arrival of the wave front. Behind the front the shear stresses are progressively reduced. The plasma is thus accelerated in the direction of the mechanical forces, and work is done on the plasma until the stored free energy is exhausted. Interaction with the magnetic forces eventually slows down this motion, and a reflected wave is sent downward from the generator switching off the stress relief motion in the transmission region. We find $j_{\|}$continues to flow upward, but the transverse component, $j_{\perp}$, reverses, thereby decreasing the integral in equation (20) until $v_{\perp}$ vanishes. In this whole region of stress relief motions, the Poynting flux is directed toward the fracture zone where it is largely absorbed.

The total time involved in this communication between fracture zone and generator is of the order of $(2-3) \times \tau_{A}$. This is the basis of equation (18).

Not all of the energy flowing into the fracture zone is, however, consumed there. The near-electrostatic nature of the field, that is, the need for return flow, requires energy to be reinvested in the magnetic field of the trailing part of the unstable current sheet. Here, however, upward and downward currents balance each other, and so do their transverse components which turn on and off the return flow, as the current sheets traverse the plasma. Inside this trailing current loop, the field is bent in the same sense as in the main stressed field region, but the flow direction shows that the stress is now applied from below, and the Poynting flux is upward. The current closes in the generator plasma and is directed parallel to the electric field, that is, the plasma there has become a load on the circuit, work has to be done to return the field, but considerably less than the energy released. This is similar to the 
work done in driving a piston back into the empty combustion chamber.

The quadrangular shape of the fracture zone is just a plausible guess. The understanding of the basic processes inside it is not well enough advanced in order to model the actual field and plasma distributions. For instance, the ratio of energy expended by heating the cool background plasma to that consumed by accelerating runaway particles is unknown. Undoubtedly, rapid heating does occur. The accompanying growth of pressure must lead to upward expansion and thus dilution of the background plasma. Thus the fracture zone becomes an "evacuation region" which digs itself into the atmosphere (Haerendel 1989). The lower border of the fracture zone in Figure 4 is meant to indicate the height decrease of a density contour. Density depletions possibly originating from this process are commonly found in the topside ionosphere (Persoon et al. 1988). The resulting variation of density and temperature through the fracture zone has an impact on the microphysics, on $c_{\text {crit }}$ and $j_{\text {crit }}$, and thus on the electric potential distribution. It should also be noted that the density depletion is counteracting the increase of thermal velocity through the heating process and thus can maintain a given current, $j_{\|}$, at the critical threshold without quenching. Much more research must be done on this subject.

Finally, there is a second wave regime between fracture zone and the current closure region near the star. Since $B$ is strong and the distances are short, Alfvén waves can travel back and forth many times within the characteristic time $\tau_{\mathrm{A}}$ as defined in equation (16). A standing wave pattern has been proposed by the author who named it "interference region" (Haerendel 1989). However, rather than a regular interference pattern nonlinear wave-wave interaction may create strong Alfvénic turbulence (Dubinin et al. 1988).

With the model contained in Figure 4 one can easily derive the variation of $B_{\perp}\left(x_{\perp}\right)$ and $E_{\perp}\left(x_{\perp}\right)$ in the upper standing wave zones if one assumes a horizontally constant magnitude of $j_{\|}$. This leads to the conclusion that one-third of the incoming Poynting flux is returning into the trailing oblique wave and that the absorbed two-thirds are properly expressed by the factor $\frac{1}{16}$ in equation (8).

\section{MICROPHYSICS}

In this paper I can only touch briefly upon the microprocesses which introduce a finite anomalous resistivity into the normally infinitely conducting plasma. Satellite measurements of the high-frequency electric field have revealed the existence of electrostatic ion-cyclotron waves (EICW) and of solitary waves, also named double layers (DL), in which the potential can jump parallel to $\boldsymbol{B}$ by a good fraction of $k T_{e}$ over scales of the order of 10 Debye lengths, $\lambda_{D}$ (Temerin et al. 1982; Boström et al. 1988). The postulated anomalous resistivity may thus be distributed (EICW) or concentrated in a multitude of small ion-acoustic double layers (DL). Both types of waves, which can coexist, may provide the enhanced electronion momentum exchange that can create the parallel potential drop, at least at those altitudes where the cool plasma of the topside ionosphere dominates the current flow.

We will evaluate one of these two microprocesses for their macroscopic consequences. The information derived from the referenced satellite experiments suggests that in the individual double layer (DL)

$$
e \Delta \phi_{\|}=o\left(k T_{e}\right)
$$

The average spacing between two DLs is of order $100 \lambda_{D}\left(\lambda_{D}=\right.$ $\left.\sqrt{k T_{e} / 4 \pi e^{2} n_{e}}\right)$. The parallel electric field averaged over many DLs would thus be

$$
\left\langle E_{\|}\right\rangle=\varepsilon \frac{k T}{e \lambda_{\mathbf{D}}}=\varepsilon \sqrt{4 \pi p_{e}}
$$

and $\varepsilon$ of order $10^{-3}$. The average number of double layers needed to produce the overall potential drop is obtained by dividing $\phi_{\|}$from equation (10) by $\left\langle E_{\|}\right\rangle$from equation (22). It is necessarily a large number.

We can define an average anomalous resistivity, $\eta_{\mathrm{an}}$, constituted by an ensemble of small ion-acoustic DL's, if we relate $\left\langle E_{\|}\right\rangle$to the critical current:

$$
\left\langle E_{\|}\right\rangle=\eta_{\text {an }} \cdot j_{\text {crit }}
$$

Introducing an effective collision frequency, $\nu^{*}$, by

$$
\eta_{\mathrm{an}}=\frac{m_{e} \nu^{*}}{e^{2} n_{e}}
$$

and using equations (1), (2), and (22), we obtain

$$
\nu^{*}=\frac{\varepsilon}{f} \cdot \sqrt{\frac{m_{i}}{2 m_{e}}} \cdot \omega_{\mathrm{pe}},
$$

where $\omega_{\text {pe }}$ is the plasma frequency and $m_{i}$ and $m_{e}$ the ion and electron masses. With $\varepsilon=O\left(10^{-3}\right)$ and $f=O(10)$, the factor in front of $\omega_{\text {pe }}$ lies between $10^{-3}$ and $10^{-2}$. For many applications this amount of accuracy is probably sufficient. The great advantage of equation (25) is that it contains solely the cold plasma density. All sophistications of the microphysics are suppressed. The effect of electrostatic ion-cyclotron waves may be taken into account by a second contribution to $\nu^{*}$ which is a fraction $(\sim 0.2)$ of the ion gyrofrequency (Lysak \& Dum 1983).

An application of equations (24) and (25) is the estimate of the macroscopic magnetic diffusivity, $D_{m}$, related to such a distributed resistivity:

$$
D_{m}=\left(\frac{c}{\omega_{\mathrm{pe}}}\right)^{2} \cdot \nu^{*}
$$

The characteristic timescale for the transverse diffusion of $\boldsymbol{B}_{\perp}$ inside the fracture zone would be

$$
\tau_{\mathrm{diff}}=\frac{w^{2}}{D_{m}} .
$$

Quantitative evaluation shows that $\tau_{\text {diff }} \ll \tau_{\text {A }}$. This means that the current and field distribution inside the fracture zone settles rapidly within the basic macroscopic timescale, $\tau_{\mathrm{A}}$. This is the origin of the near-electrostatic nature of the field. Since $\tau_{\text {diff }}$ 
is also the timescale for electromagnetic energy conversion and $c_{\mathrm{AF}}$ is the speed of magnetic energy supply from above the fracture zone,

$$
\ell_{\|}=c_{\mathrm{AF}} \cdot \tau_{\mathrm{diff}}
$$

must give an estimate of the height extent of the energy conversion region or fracture zone. Outside the fracture zone, where the current is stable, $D_{m} \cong 0$.

At this point, I like to draw attention to an important aspect of this theory. Only the existence of some sort of anomalous resitivity is postulated in order to justify the appearance of a field-aligned potential drop. Its magnitude is derived from other considerations ( see $\S 2$ ). One sees that $\nu^{*}$ and $\eta_{\text {an }}$ determine solely the height distribution of $\phi_{\|}$, that is, $\left\langle E_{\|}\right\rangle$and $\ell_{\|}$. Thus the latter is the only key parameter following from microscopic rather than macroscopic considerations.

\section{APPLICATION TO SOLAR FLARES}

The perhaps strongest observational hint to the existence of electrostatic acceleration during flares comes from those (few) events of very short duration ( $\leq 10 \mathrm{~s}$ ) which have gamma-ray spectra showing flat continua between 0.3 and $9 \mathrm{MeV}$ with negligible contributions from nuclear lines (Rieger \& Marschhäuser 1990). The extreme temporal variation suggests an acceleration process that does not favor ions, like shock wave acceleration does. An electrostatic field-aligned process would fulfill this requirement. However, other processes simultaneously accelerating ions and electrons have been proposed (e.g., Sakai \& Ohsawa 1987). In this section I just want to demonstrate that the outlined theory, if applied to the solar flare situation, is capable of accounting for the electrons necessary to produce these gamma events. Other examples can be found in Haerendel (1989).

The short duration of the above-mentioned events seems to preclude a stationary current configuration for the energy conversion process. It is more likely that the required shear stresses are applied to the magnetic field in the corona in the process of violent dissipation and magnetic reconfiguration. It has become increasingly popular (Haerendel 1987; Parker 1987; Vlahos 1989) to think of the energy storage in active regions in terms of a great number of current filaments. Figure 5 sketches such a configuration, except that in reality the number of small-scale structures may be several orders of magnitude higher. A flare may be initiated by a large-scale change of the established equilibrium, for example, by instability, coronal mass ejection or emergence of new magnetic flux. Rapid dissipation in a multitude of narrow current sheets would follow and constitute the main energy release process (Parker 1987). One very likely consequence would be that in this process strong transient magnetic shears would be generated, for example, via braking of narrow reconnection flows. These shear stresses could carry energy out of the primary dissipation region in the corona toward the chromosphere. In hitting the steep density gradient at about $2000 \mathrm{~km}$ altitude, an Alfvén wave would be reflected. The reflection enhances $j_{\Downarrow}$ and $B_{\perp}$ by about a factor of 2 . Thus the mapping relation of equation (14a) would read:
HUNDRED THOUSAND CAVITIES AS BIRTHPLACES OF HIGH ENERGY PARTICLES AND GAMMA RAYS

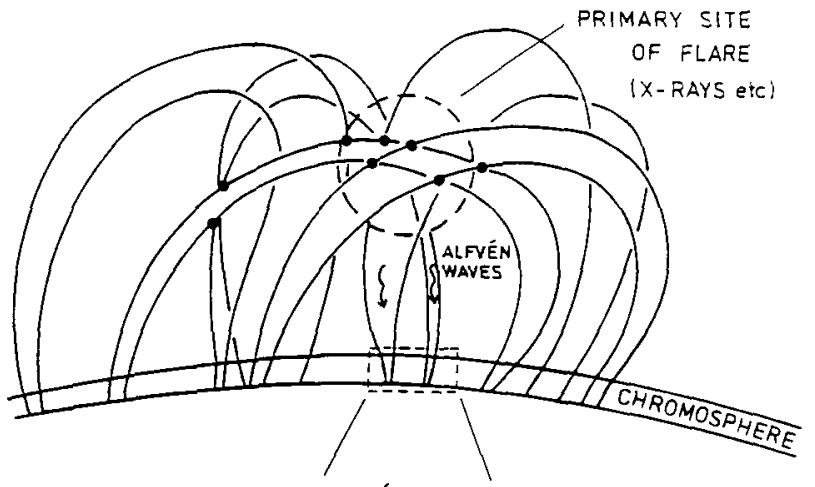

ALFVÉN WAVES

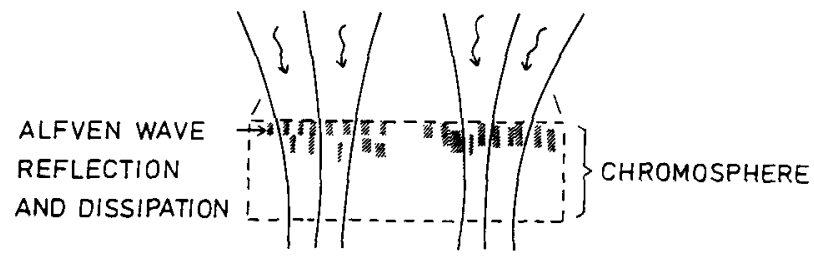

FIG. 5.-Solar flare scenario that may lead to a large number of parallel potential drops in the topside chromosphere. The primary energy release process is thought to occur inside the corona in a multitude of reconnection processes between adjacent sheared or twisted flux tubes. A fraction of the released energy can be reinvested in setting up new magnetic shear stresses, of which a small fraction may be sufficiently strong and concentrated to generate supercritical field-aligned currents in the chromosphere and thus establish the conditions for field-aligned potential drops.

$$
\Delta B_{\perp \mathrm{chr}}=2\left(\frac{\rho_{\mathrm{chr}}}{\rho_{\mathrm{cor}}}\right)^{1 / 4} \Delta B_{\perp \mathrm{cor}} ;
$$

$\rho_{\text {chr }} / \rho_{\text {cor }} \cong 10^{2}$ is a reasonable value.

On top of the chromosphere, the electron density is of order $3 \times 10^{10} \mathrm{~cm}^{-3}$ and $T_{e} \cong 8000 \mathrm{~K}$. These numbers and $f=10$ in equation (2) yield a critical current of $\sim 400 \mathrm{~A} / \mathrm{m}^{2}$. The typical width of an unstable current sheet follows from equations (3) and (29):

$$
2 w=60 m \cdot\left(\frac{\Delta B_{\perp \text { cor }}}{10^{2} \mathrm{G}}\right)
$$

The high atmospheric density requires very high current density and thus strong spatial concentration in sheets of the order of tens of meters in order for $j_{1}$ to reach the instability threshold. However, this is no principal difficulty, since the energy release by interacting current tubes in the hot corona, which constitutes the generator in this flare scenario (see Haerendel 1987), also requires spatial scales of $10 \mathrm{~m}$ or less (Parker 1987).

The possible values of $\phi_{\|}$developing in a reflected Alfvén wave under current instability follow from equation (10):

$$
\phi_{\|}=5 \mathrm{MV} \cdot\left(\frac{B_{\mathrm{chr}}}{10^{3} \mathrm{G}}\right) \cdot\left(\frac{\Delta B_{\perp \mathrm{cor}}}{10^{2} \mathrm{G}}\right)^{2} \text {. }
$$


This result allows two conclusions: (1) The discussed process can generate megavolt field-aligned potential drops, and (2) rather strong primary fields are required in order to reach values of $\phi_{1}$ of up to $60 \mathrm{MV}$ which some gamma-ray spectra imply (Rieger \& Marschhäuser 1990). On the other hand, $\phi_{\mathbf{l}}$ in equations (10) and (31) is not the peak value in the acceleration region, but an average.

If we now turn to the energy conversion rate $\dot{\varepsilon}_{1}$, we find

$$
\dot{\varepsilon}_{\|}=2 \times 10^{12} \mathrm{ergs} \mathrm{cm}^{-2} \mathrm{~s}^{-1} \cdot\left(\frac{B_{\mathrm{chr}}}{10^{3} \mathrm{G}}\right)\left(\frac{B_{\perp \mathrm{cor}}}{10^{2} \mathrm{G}}\right)^{2} .
$$

In a current sheet of width, $2 w$, and maybe 10 times greater length, the energy conversion per unit time is (see eq. [30]):

$$
\begin{aligned}
\dot{W} & =\dot{\varepsilon}_{\|} \times 10 w^{2} \\
& =7.2 \times 10^{20} \mathrm{ergs} \mathrm{s}^{-1} \cdot\left(\frac{B_{\text {chr }}}{10^{3} \mathrm{G}}\right)\left(\frac{B_{\perp \text { cor }}}{10^{2} \mathrm{G}}\right)^{4} .
\end{aligned}
$$

No more than about $10^{26} \mathrm{ergs}^{-1}$ in $\mathrm{MeV}$ electrons are needed to explain the gamma-ray fluxes. This means that on the order of $10^{5}$ (or less) unstable current sheets, which would fill only $10^{-6}$ of a typical flare area, would suffice to produce the observed gamma-ray bursts. In order to account for the observed temporal variations of the order of $1 \mathrm{~s}$, we suspect that this type of energy release does not occur throughout the flare area, but in a small fraction of it. If the magnetoacoustic speed is taken as communication speed in triggering the considered Alfvén waves, one can conclude that as little as $1 \%$ of the flare area is responsible for the gamma-ray emissions. Even then the filling factor by fracture zones would only be $10^{-4}$. Hence, a great many of field-aligned potential drops in the upper chromosphere are serious candidates for the sites of $\gg 1 \mathrm{MeV}$ electron and ion beam generation.

The scope of this paper does not permit us to go into any further detail on this topic. It is meant as an illustration for the power of the magnetic fracture process to produce dense energetic particle beams in stellar environments which, by virtue of their high energy, produce easily detectable electromagnetic signals. The structure of equations $(9)$ and ( 10$)$ shows readily that more strongly magnetized stars than our Sun would produce much higher energies and energy fluxes.

\section{CONCLUSIONS}

The paper is an attempt to demonstrate that magnetic energy release by field-aligned potential drops in low-beta plasmas is probably a common process in stellar magnetic fields. Necessary ingredients are (1) the application of shear stresses on the field by external forces and (2) instability of the attending field-aligned currents somewhere along the circuit, mostly in a plasma of very low beta value. The region of current instability is normally widely separated from the region where the shearing forces are applied (high beta). In any astrophysical situation it is difficult to predict whether the currents will be sufficiently concentrated in order to reach the critical threshold for instability. However, the most likely location of such instability inside the current circuit transporting the shear stresses can be predicted, if the structure of the outer stellar atmosphere and magnetic field is known. The theory allows evaluation of the magnitude of the potential drops, the energy conversion rate, and the spatial dimensions, in case current instability and formation of parallel potential drops do occur. For practically all cosmical objects except our planetary system, the spatial scales of the individual potential drop (fracture zone) are too narrow to be resolvable by any remote sensing technique. One has to estimate further the density of occurrence of such potential drops. This may be achieved from the knowledge of the total energy released in form of particle beams which manifest themselves by some kind of electromagnetic bremsstrahlung.

The presented process of converting stored magnetic energy into kinetic energy of particles is very powerful in two respects. Extremely high individual particle energies (Haerendel 1989) can be reached in a one-step electrostatic acceleration from thermal energies. No seed particles, no storage volumes are needed. Second, the conversion efficiency is very high, up to $\frac{2}{3}$. Therefore, high-luminosity signals at $\gamma$-ray energies are to be expected from stellar systems with strong magnetic field subject to some kind of strong interaction with companion stars, accretion disks, interstellar medium, or trapped plasmas. These signals bear information on the dynamical processes leading to the underlying magnetic shear stresses and on the location and properties of the energy conversion plasma.

\section{REFERENCES}

Alfvén, H., \& Fälthammar, C.-G. 1963, Cosmical Electrodynamics, Fundamental Principles (Oxford: Oxford Univ. Press)

Borovsky, J. E. 1993, J. Geophys. Res., 98, 6101

Boström, R., Gustafsson, G., Holback, B., Holmgren, G., Koskinen, H., \& Kintner, P. 1988, Phys. Rev. Lett., 61, 82

Casserly, R. T., \& Cloutier, P. A. 1975, J. Geophys. Res., 80, 2165

Colgate, S. 1978, ApJ, 221, 1068

Dubinin, E. M., Volokitin, A. A., Israelivich, P. L., \& Nikalaeva, N. S. 1988, Planet. Space Sci., 36, 949

Fridman, M., \& Lemaire, J. 1980, J. Geophys. Res., 85, 664

Goldreich, P., \& Julian, W. H. 1969, ApJ, 157, 869

Gorney, D. J., Clarke, A., Croley, D. R., Fennell, J. F., Luhmann, J. M., \& Mizera, P. F. 1981, J. Geophys. Res., 86, 83

Gurnett, D. A. 1972 in Critical Problems of Magnetospheric Physics, ed. E. R. Dyer (Washington: Natl. Acad. Sci.), 123
Haerendel, G. 1980, ESA J., 4, 197

- 1987, Proc. 2 I st ESLAB Symp., Bolkesjø, Norway (ESA SP-275), 205

1989, Proc. Varenna-Abastumani Intern. School \& Workshop on Plasma Astrophysics, Varenna 1988 (ESA SP-285), vol. I, 37

Haerendel, G., Buchert, S., LaHoz, C., Raaf, B., \& Rieger, E. 1993, J. Geophys. Res., 98, 6087

Hallinan, T. J., \& Davis, T. N. 1970, Planet. Space Sci., 18, 1735

lijima, T., \& Potemra, T. A. 1976, J. Geophys. Res., 81, 5971

Kindel, J. M., \& Kennel, C. F. 1971, J. Geophys. Res., 76, 3055

Knight, S. 1973, Planet. Space Sci., 21, 741

Lyons, L. R. 1980, J. Geophys. Res., 85, 17

Lysak, R. L., \& Dum, C. T. 1983, J. Geophys. Res., 88, 365

Mozer, F. S., Carlson, C. W., Hudson, M. K., Torbert, R. B., Parady, B., Yatteau, J., \& Kelly, M. C. 1977, Phys. Rev. Lett., 38, 292 


\section{HAERENDEL}

Papadopoulos, K. 1977, Rev. Geophys. Space Phys., 15, 113

Parker, E. N. 1987, Sol. Phys., 111, 297

Persoon, A. M., Gurnett, D. A., Petersen, W. K., Waite, J. H., Burch, J. L., \& Green, J. L. 1988, J. Geophys. Res., 93, 1871

Rieger, E., \& Marschäuser, H. 1990, Proc. MAX 91 Workshop no. 3, ed. R. M. Winglee \& A. L. Kiplinger (Boulder: Univ, of Colorado Press), 68 Sakai, J.-I., \& Ohsawa, Y. 1987, Space Sci. Rev., 46, 113

Sharp, R. D., Johnson, R. G., \& Shelley, E. G. 1977, J. Geophys. Res., 82, 3324
Temerin, M., Cerny, K., Lotko, W., \& Mozer, F. S. 1982, Phys. Rev. Lett., 48,1175

Vasyliunas, V. M. 1975, Rev. Geophys. Space Phys., 13, 303

Vlahos, L. 1989, Sol. Phys., 121, 431

Wescott, E. M., Stenbaek-Nielsen, H. C., Hallinan, T. J., \& Davis, T. N. 1976, J. Geophys. Res., 81, 4495 